\title{
Uber die Konvergenz eines mit einer Potenzreihe assoziierten Kettenbruchs ${ }^{2}$ ).
}

\section{Von}

Hans Hamburger in Berlin.

1. Es sei $z=x+i y$ eine komplexe Veränderliche und

$$
S(z)=\frac{c_{0}}{z}+\frac{c_{1}}{z^{2}}+\ldots
$$

eine beliebige formale Potenzreihe, von der nur vorausgesetzt werde, daß die aus ihron Koeffizienten $c_{v}$ gebildeten Hankelschen Determinanten

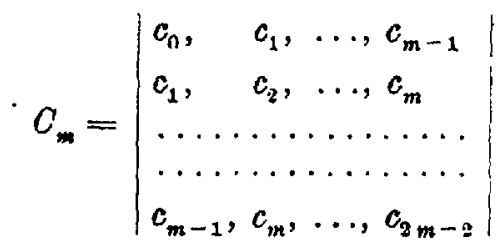

für alle $m$ von Null verschieden sind. Uber die Konvergenzeigenschaften von $S(z)$ werden hier keine weiteren Annahmen gemacht, so da $B$ also auch solche Reihen $S(z)$ in den Kreis unserer Betrachtungen einbezogen werden, die für jeden noch so groben Wert des absoluten Betrages von z divergent sind.

Nach bekannten Sätzen läßt sich dann durch einen formalen Divisions-. prozeß die Reihe $S(z)$ in einen Kettenbruch

$$
K(z)=\frac{c_{1}}{z+\beta_{1}}+\frac{\alpha_{9}}{z+\beta_{2}}+\frac{c_{3}}{z+\beta_{8}}+.
$$

1) Den in dieser Arbeit veröffentlichten Satz habe ioh ip̣ Frâhjahr 1916 gefunden. Duroh militärische Verpflichtungen gehindert, konnte ich dés Manuskript. erst im März 1917 fertigstellen und sandte es denn bald darauf an die Redaktion des Journals fut reine und angewandte Mathematik, die dio Arbeit annahm. In Februar 1919 habo ioh das Manuekript zurïokerbeten, da ioh orkannt batte, dab sich mein ursprünglicher Beweis, wesentlioh vereinfachen ließ. 
entwickeln ${ }^{2}$ ), den wir mit Herrn Perron mit der Potenzreihe $\boldsymbol{S}(z)$ assoziiert

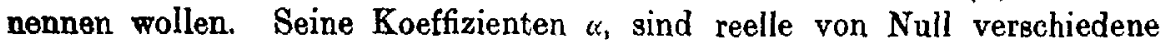
Zahlén, seiné Koeffizienten $\beta_{r}$, beliebige reelle Zahlen.

Der $n$-te Näherungsbruch $K_{n}(z)$ des Kettenbruchs $K(z)$ ist, wie sich sus den Rekursionsformeln für Kettenbrüche unmittelbar ergibt, gleich dem Quotienten zweier Polynome

$$
K_{n}(z)=-\frac{U_{n}(z)}{\Gamma_{n}(z)},
$$

wobei $U_{n}(z)$ vom Grade $n-1, V_{n}(z)$ vom Grade $n$ ist; außerdem ist in $\nabla_{n}(z)$ der Koeffizient des Gliedes $z^{n}$ gleich 1 .

Offenbar läßt sich $K_{n}(z)$ für hinreichend große Werte von $z$ in eine konvergente Potenzreihe entwickeln:

$$
K_{n}(z)=-\sum_{\nu=0}^{\infty} \frac{c_{v}^{(n)}}{z^{n+1}} .
$$

Nunmehr zeigt sich der Zusammenhang zwischen der Potenzreihe und dem mit ihr assozilerten Kettenbruch darin, daß die $2 \boldsymbol{n}$ ersten Koeffizienten der Potenzreihenentwicklung von $K_{n}(z)$ mit den entsprechenden Koeffizienten ,von $S(z)$. übereinstimmen, daß also die Beziehungen bestehen ${ }^{3}$ )

$$
c_{y}^{(n)}=c_{r} \text {. für } y^{\prime}=0,1, \ldots, 2 n-1 .
$$

Durch den Grad der Polynome $U_{n}(z)$ und $V_{n}(z)$ und durch die Beziehungen (2) ist die rationsle Funktion $K_{n}(z)$ eindeutig bestimmt.

Die Bedingung $C_{m} \neq 0$ für $m=0,1, \ldots, n-1$ erweist sich ferner nicht nur als hinreichend, sondern auch als notwendig dafür, daß eine Funktion $K_{n}(z)$ von den verlangten Eigenschaften existiert. Dieses Resultat läBt sich auch in dein Satze formulieren: Damit der mit einer Potenzreihe $S(z)$ sssoziierte unendliche Kettenbruch $K(z)$ existiert, ist notwendig und hinreichend, daB die sus den Koeffizienten $c_{y}$ der Potenzreihe gebildeten Determinanten $C_{m}$ für alle Werte von $m$ von Null verschieden $\sin d^{8}$ ).

Umgekehrt läßt sich zu jedem Kettenbruch $K(z)$ der Form (1) eine nit ihm assoziierte Potenzreihe $S(z)$ finden, deren Koeffizienten $z u$ den Koeffizienten der Potenzreihenentwicklung von $K_{n}(z)$ in den durch Formel (2) angegebenen Beziehungen stehen ${ }^{8}$ ).

3) O. Perron, Die Lehre ron den Kettenbrüohen, im folgenden kurz mit Perron, Lebrbuch zitiert. Leipzig 1913. S. 322-326 und S. 376. Sieh'e auoh J. Grommer, Ganze transzendente Funktionen mit lauter reellen Nullstellen, Dissertation Göttingen 1914, abgèdruokt im Journal für reine und angewandte Math. 14t (1014), S. 114-165, im folgenden kurz mit Grommer, Dissertation zitiert. Vgl. insbesondere S. 118-123.

') J. c. Anm. "). 
2. Besonderes Interesse verdienen diejenigen Kettenbrüche, deren Koeffizienten $\alpha_{\nu}$ sämtlich negative Zablen sind mit Ausnahme von $\alpha_{3}$, das größer als Null ist. Sie sind mit solchen Potenzreihen $S(z)$ assoziiert, deren Koeffizientendeterminanten $C_{m}$ sämtlich positiv sind. Aus der Bedingung $C_{m}>0$ für alle $m$ folgt leicht $c_{2},>0$, während die Koeffizienten $c_{3 v+1}$ positiv oder negativ oder auch gleich Null sein können.

Die Bedingung $C_{m}>0$ für alle $m$ ist notwendig und hinreichend dafür, daß $a_{1}>0, \alpha_{\nu}<0$ für $v \geqq 2$ wird. Im folgenden sollen nur Kettenbrüche $K(z)$ mit dieser Koeffizienteneigenschaft betrachtet werden.

Ihre wichtigste Eigenschaft zeigt sich bei der Partialbruchzerlegung der Näherungsbrüche $\left.K_{n}(z)^{4}\right)$. Es ergibt sich nämlich

$$
K_{n}(z)=\sum_{x=1}^{n} \frac{M_{x}^{(n)}}{z-\lambda_{x}^{(n)}} .
$$

Hierbei sind die $\lambda_{x}^{(n)}$, die Nullstellen des Polynoms $V_{n}(z)$, sämtlich reell und voneinsnder verschieden, die Residuen von $K_{n}(z)$

sämtlich positiv.

$$
M_{*}^{(n)}=\frac{V_{n}\left(\lambda_{x}^{(n)}\right)}{V_{n}^{\prime}\left(\lambda_{x}^{(n)}\right)}
$$

3. In seiner Arbeit über ganze transzendente Funktionen mit lauter reellen Nullstellen untersucht Herr Grommer Kettenbrüche der eben bèschriebenen Art, die mit für $|z|>\varrho$ konvergenten Potenzreihen assoziiert sind, und beweist dort den Satz ${ }^{5}$ ):

Ist der Kettenbruch $K(z)$ mit einer Potenzreihe $S(z)$ assoziiert, deren Koeffizientendeterminanten $C_{m}$ sämtlich positiv sind und die für $! z \mid>\varrho$ konvergiert, so konvergiert der Kettenbruch $K(z)$ gleichmäßig in jedem abgeschlossenen Bereich der $z$-Ebene, dem kein Punkt der reellen Achse, der zwischen $-\varrho$ und $+\varrho$ gelegen ist, angehört, und stellt dort dieșelbe analytische Funktion $f(z)$ wie, für $|z|>\varrho$, die Potenzreihe $S(z)$ dar.

4. In der vorliegenden Arbeit soll ein etwas allgemeinerer $\$ \int^{6}$ selben Art bewiesen werden, indem die Vorqussetzung, daß dió Pótenzreike $S(z)$ einen Konvergenzkreis besitzt, abgeändert wird. Der zu beweisende Satz lautet:

IIs sei eine formale Potenzreihe $\mathcal{S}(z)$ vorgelegt, deren 'Koéffizientendeterminanten $C_{m}$ sämtlich positiv sind. Außerdem mogen žuved Konstanten

4) Vgl. etwa Grommer, Diss. S. 123-125.

b) Dies Isap. I, 8. 129-134. 
$\varrho$ und $k$ von der Beschaffenheit existieren, da $\beta$ die Koeffizienten $c_{\nu}$ der Potenzreihe $\mathbb{S}(z)$ sich durch die Beziehung

abschätzen lassen.

$$
\left|c_{r}\right| \leqq \frac{k}{\varrho^{p}} y !
$$

Dann konvergient der Kettenbruch $K(z)$ gleichmäßig in jedem abgeschlossenen Bereich der z-Ebene, der kein Stïck der Achse der reellen Zahlen enthail.

Durch den Kettenbruch $K(z)$ wird also in der Halbebene mit positivem Imaginärteil eine analytiache Funktion $f(z)$, in der Halbebene mit negativem Imaginärteil eine analytische Funktion $f^{*}(z)$ definiert, und zwar ist

$$
f^{*}(x-i y)=\bar{f}(x+i y),
$$

wenn $\bar{f}$ den zu $f$ konjugiert komplexen Wort bezeichnet. Die Funktíon $f^{*}(z)$ wird aber im allgemeinsten Falle nicht die analytischo Fout setzung von $f(z)$ sein, da z. B. die Funktion $f(z)$ die Achse der reellen Zahlen als natürliche Grenze haben kann.

Für die Funktion $f(z)$ wird auBerdem die Integraldarstellung

$$
f(z)=\int_{0}^{-i \infty} v(t) e^{-t z} d t=-i \int_{0}^{\infty} v(-i \tau) e^{i+z} d \tau
$$

gefunden, wobei $v(t)$ sich in die wegen (3) für $\mid t_{1}<\varrho$ konvergente Potenzreibo

$$
v(t)=\sum_{\nu=1}^{\infty} \frac{c_{\nu}}{\nu !} t^{\nu}
$$

entwickeln läBt. Das heiBt aber nichts anderes, als daß sich die Funktion $f(x)$ auch durch Borelsche summation aus der. Potenzreihe $S(z)$ ergibt; da außerdem das Integral rechter Hand von (4) sich für $y \geqq \delta$, unter $\delta$ eine beliebig kleine positive Zahl verstanden, als absolut und gleichmäßig konvergent erweist, so ist die im allgemeinen divergente Potenzreibe $S(z)$ im Borelschen Sinne sogar absolut summierbar.

Aus der Integraldarstellung (4) folgt ferner nach bekanntea Sätzen von Poincaré ${ }^{\circ}$ ) and Herrn Borel, daB die Funktion $f(z)$ im Winkelraum $\delta \leqq$ arg $z \leqq \pi-\delta$ durch die Potenzreihe $S(z)$ im Poincaréschen Ginpe asymptotisch dargestellt wird.

5. Der Beweis des Konvergenzsatzes berubt im wesentlichen auf eing funktionentheoretischen Hilfssatze, der auch für aich selbst von Intexdog erscheint:

6) H. Poincaré, Sur les intégrales irrégulières des équations linéaires, susk Math. 8 (1886), S. 295-344. Vgl. insbesondere S. 295-296 und S. 807-312: 
Ist $t=\sigma+i \tau$ eine komplexe Veränderliche und ist

$$
v(t)=\sum_{\nu=0}^{\infty} b_{v} t^{\nu}=\sum_{\nu=0}^{\infty} \frac{c_{v}}{v !} t^{\nu}
$$

eine für $|t|<\varrho$ konvergente Potenzreihe, sind endlich die Koeffizientendeterminanten

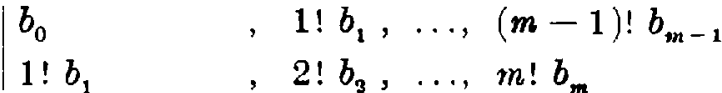

$$
\begin{aligned}
& \left|\begin{array}{c}
\ldots \ldots \ldots \ldots \ldots \ldots \ldots \ldots \ldots \ldots \ldots \ldots \ldots \ldots \ldots \ldots \ldots \\
\ldots \ldots \ldots \ldots \ldots \ldots \ldots \\
(m-1) ! b_{m-1}, \quad m ! b_{m}, \ldots,(2 m-2) ! b_{2 m-2}
\end{array}\right|=C_{m}
\end{aligned}
$$

sämtlich positiv, so läßt sich die Funktion $v(t)$ im ganzen Streifen $-\varrho+\delta \leqq \sigma \leqq \varrho-\delta$ fortsetzen und bleibt dort unterhalb einer festen, von $t$ unabhängigen Schranke.

Der Beweis dieses Satzes wird in $\S 2$ geführt und enthält den wesentlichen mathematischen Gedanken der Arbeit.

Im $\$ 3$ wird der Bew.eis der Konvergenz des Kettenbruchs zu Ende geführt, der nun keine Schwierigkeiten mehr macht.

Im $\$ 4$ endlich werden für die Funktion $f(z)$ noch Darstellungen durch Stieltjessche Integrale und durch Fakultätenreihen kurz angegeben.

6. Die in den ersten drei Paragraphen ausgeführten Beweise tragen elementaren Charakter und benutzen vor allen Dingen nicht die Darstellung der Koeffizienten $c_{y}$ durch Stieltjessche Integrale:

$$
c_{v}=\int_{-\infty}^{+\infty} u^{v} d \Phi(u)
$$

Die Existenz einer solchen Darstellung ist unter Benutzung des Grommer: schen Auswahltheorems ${ }^{i}$ ), das das Differential $d \Phi(u)$ liefert, in dem allgemeinen Falle, der hier in Frage kommt, vom Verfasser zum ersten Male nachgewiesen worden, welcher unter Benutzung dieser Darstellung die Konvergenz des Kettenbruchs $K(z)$ auch an anderer Stelle bewiesen hat ${ }^{8}$ ). Ebenda sind die Resultate anderer Arbeiten ${ }^{\circ}$ ) über denselben Gegentiond angeführt, die auch von der Integraldarstellung (6) der Koeffizienten $c_{r}$ Gebrauch machen.

7) Diss. Kap. III, S. 184-145.

8) H. Hamburger, Beiträge zur Korvergeaztheoris der Stieltjessohen Ketten-

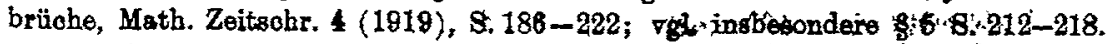

) O. Perron, Erweiterung eines Markoffechen-Satzes über-die Konvergenz ge-

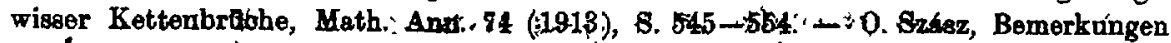

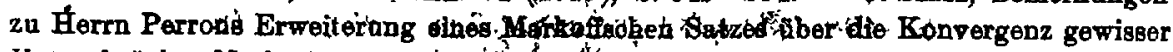
Kettenbrüche, Math. Ann. 76 (4913), $801-814$. 
Der funktionentheoretische Satz über die Fortsetzbarkeit der analytischen Funktion $v(t)$ ist unter Benutzung der Integraldarstellung (6) vom Verfasser gleichfalls an anderer Stelle ${ }^{10}$ ) bewiesen und als eine Art von Umkehrung mit einem Satze von Herrn Pólya ${ }^{11}$ ) über die Funktionen $\frac{1}{g(z)}$, wo $g(z)$ eine ganze transzendente Funktion mit nur reellen Nullstellen vom Geschlechte 0 oder 1 bezeichnet, in Zusammenhang gebracht worden. Doch glaube ich, daß trotzdem die hier ausgeführten elementaren Betrachtungen einiges Interesse verdienen.

\section{$\S 1$.}

Es sei eine Potenzreihe $S(z)$ vorgelegt, deren Koeffizientendeterminanten $C_{m}$ sämátlich positiv sind und deren Koeffizienten $c_{\nu}$ der Ungleichheitsbeziehung (3) genügen.

Wie in der Einleitung auseinandergesetzt wurde, existiert dann der mit $S(z)$ assoziierte Kettenbruch $K(z)$, und zwar wird $\alpha_{1}>0, \alpha_{v}<0$ fiùr $v \geq 2$.

Für seinẹ Näherungsbruch $n$-ter Ordnung $K_{n}(z)$ erhält man die Darstellungen

$$
K_{n}(z)=\frac{U_{n}(z)}{\nabla_{n}(z)}=\sum_{x=1}^{n} \frac{M_{x}^{(n)}}{z-\lambda_{x}^{(n)}}=\sum_{\nu=0}^{\infty} \frac{c_{v}^{(n)}}{z^{p+1}},
$$

wobei für die Koeffizienten $c_{y}^{(n)}$ die fundamentalen Beziehungen (2) gelten.

Die Partialbruchżerlegung für $K_{n}(z)$ wollen wir jetzt in anderer Form schreiben, indem wir $\frac{1}{z-\lambda_{\varkappa}^{(n)}}$ durch das für $y \geqq \delta$ absolut und gleichmäßig konvergente Integral $\int_{0}^{-i \infty} e^{-t\left(z-\lambda_{\%}^{(t)}\right)} d t$ ersetzen. Es ergibt sich so

wenn

gesetzt wird.

$$
K_{n}(z)=\sum_{x=1}^{n} M_{x}^{(n)} \int_{0}^{-i \infty} e^{-t\left(z-\lambda_{z}^{(n)}\right)} d t=\int_{0}^{-i \infty} v_{n}(t) e^{-t z} d \dot{t}
$$

$\left.{ }^{10}\right)$ H. Hamburger, Bemerkungen za einer Fragestellung des Herm Pólya; wird in der Math. Zeitschr, ersoheinen.

11) G. Pólya, Algebraische Untersuchungen über ganze Funktionen rom Geschlechte 0 und 1, Journal für reine und angew. Math. 14.5 (1915), S. 224-249, "vgl. insbes. S. 235-237. 
Da $v_{n}(t)$ eine ganze transzendente Funktion ist, läßt sich $v(t)$ an der Stelle $t=0$ in eine Potenzreihe

$$
v_{n}(t)==\sum_{r=0}^{\infty} b_{r}^{(n)} t^{\prime \prime}
$$

entwickeln, und zwar erhält man wegen (9)

$$
b_{r}^{(n)}=\frac{1}{\nu !} \sum_{x=1}^{n} M_{x}^{(n)}\left(\lambda_{x}^{(n)}\right)^{\nu} .
$$

Aus (7) folgt aber andererseits

also

$$
\sum_{x=1}^{n} M_{x}^{(n)}\left(\lambda_{x}^{(n)}\right)^{\nu}=c_{\nu}^{(n)},
$$

$$
b_{y}^{(n)}=\frac{c_{y}^{(n)}}{\nu !} .
$$

Wir beweisen zunächst, daß für $|t| \leqq \varrho-\delta$, unter $\delta$ eine beliebig kleine positive Zahl verstanden, die Funktionen $v_{n}(t)$ mit wachsendem $n$ gleichmäßig gegen die Funktion

$$
v(t)=\sum_{\nu=0}^{\infty} b_{\nu} t^{\nu}=\sum_{\nu=0}^{\infty} \frac{c_{\nu}}{\nu !} t^{\nu}
$$

konvergieren. $\mathrm{Da} ß$ die Potenzreihe (11) für $|t|<\varrho$ konvergiert, folgt unmittelbar aus der Absohätzung (3) für $\left|c_{\nu}\right|$.

Gelegentlich des Beweises seines in der Einleitung zitierten Konvergenzsatzes zeigt Herr Grommer, da.B

$$
c_{2}^{(n)} \leqq c_{2 \nu}
$$

ist für alle $n$ und alle $\nu$. Der Nachweis dieser Ungleichheitsbeziehung, den wir der Vollständigkeit halber hier kurz skizzieren wollen, stützt sich auf einen Hilfssatz, der in größerer Allgemeinheit von Laguerre ${ }^{12}$ ) formaliert worden ist, und für den in der hier erforderlichen speziellen Fassung Herr Grommer einen einfachen algebraischen Beweis angibt. Der Satz lautet:

Es sei $P(z)$ ein Polynom $m$-ten Grades mit nur reellen Nállstellen. Außerdem sei der Koeffizient des Gliedes $z^{m}$ gleioh 1 . Entwickelt man $\frac{1}{P(z)}$ in die Potenzreihe

$$
\frac{1}{P(z)}=\frac{a_{0}}{z^{m}}+\frac{a_{1}}{z^{m+1}}+\frac{a_{z}}{z^{m+2}}+\ldots
$$

so sind alle Koeffizienten $a_{2}>0$.

19) Laguerre, Guî̀res, Bd. I, 8. 169 ; Grommé, Dise, 8. 180-132. 
Aus diesem Hilfssatz folgert Herr Grommer die Beziehung $c_{2 v}^{(n)} \leqq c_{21}$, indem er von der Fundamentalformel für Kettenbrüche ${ }^{{ }^{3}}$ )

$$
\frac{U_{n+1}(z)}{\bar{V}_{n+1}(z)}-\frac{U_{n}(z)}{\bar{V}_{n}(z)}=\frac{(-1)^{n} \alpha_{1} \alpha_{2} \ldots \alpha_{n+1}}{\bar{V}_{n+1}(z) V_{n}(z)}
$$

Gebrauch macht. Entwickelt man nämlich $\frac{1}{\widetilde{V}_{n+1}(z) V_{n}(z)}$ in eine Potenzreihe $\frac{1}{z^{2 n}} \sum_{v=0}^{\infty} \frac{a_{v}}{z^{\nu+1}}$, so ist $a_{3 v}>0$, da das Polynom $V_{n+1}(z) V_{n}(z)$ allen Voraussetzungen des Laguerreschen Hilfssatzes genügt. Andererseits folgt aber aus (12), indem man die Potenzreibenentwicklung (7) der Näherungsbrüche $K_{n}(z)$ berücksichtigt,

$$
c_{2 v}^{(n+1)}-c_{2 r}^{(n)}=(-1)^{n} c_{1} c_{2} \ldots u_{n+1} a_{2 n-2 n}
$$

für $v \geqq n$; also, da außerdem $\alpha_{1}>0,-c_{2}>0, \ldots,-\alpha_{n+1}>0$ ist,

$$
c_{2 \nu}^{(n+1)}-c_{2 v}^{(n)}>0 \text { für } \nu \geqq n \text {. }
$$

Nun ist aber nach Formel (2) der Einleitung

$$
c_{2 \nu}^{(n+m)}=c_{3} \text {, für } n+m \geqq \nu+1 \text {, }
$$

also, mit Rücksicht auf die Abschätzung (3)

$$
c_{3^{2}}^{(n)} \leqq c_{2 v}^{(n+1)} \leqq \cdots \leqq c_{2 \nu}^{(n+m)} \leqq c_{2 \nu} \leqq \frac{k}{e^{2 v}}(2 \nu) !,
$$

wie behauptet wurde.

Aus der Summendarstellung (10) für die Koeffizienten $c_{v}^{(n)}$ folgt ferner, daß die quadratische Form

$$
c_{2 \nu}^{(n)} x_{0}^{2}+2 e_{2 \nu+1}^{(n)} x_{0} x_{1}+c_{2 \nu+2}^{(n)} x_{1}^{2}=\sum_{x=1}^{n} M_{x}^{(n)}\left(x_{0}\left(\lambda_{x}^{(n)}\right)^{\nu}+x_{1}\left(\lambda_{x}^{(n)}\right)^{\nu+1}\right)^{2}
$$

positiv definit, und damit ihre Determinante

$$
c_{2 v}^{(n)} c_{2 v+2}^{(n)}-\left(c_{2 v+1}^{(n)}\right)^{2}>0
$$

ist. Dies ergibt aber für $c_{2 x+1}^{(n)}$ die Abschätzung

(14) $\left|c_{2 v+1}^{(n)}\right| \leqq \sqrt{c_{2 v}^{(n)} c_{2 v+2}^{(n)}} \leqq \sqrt{c_{2 v} c_{2 v+s}}$

$$
\leqq k \sqrt{1+\frac{1}{2 v+1}}\left(\frac{1}{\varrho}\right)^{2 v+1}(2 v+1) ! \leqq k \sqrt{2}\left(\frac{1}{\varrho}\right)^{2 v+1}(2 v+1) !,
$$

wenn man die Abschätzung (13) für die $c_{2 \nu}^{(n)}$ berücksichtigt.

Wegen (2) wird nun aber

$$
v(t)-v_{n}(t)=\sum_{\nu=2 n}^{\infty} \frac{c_{v}-c_{\nu}^{(n)}}{\nu !} t^{\nu}
$$

23) Vgl. Perron, Lehrbuch, S. 16 u. 378. 
also erhält man für $|t| \leqq \varrho-\delta$, indem man die Abschätzungen (3), (13) und (14) benutzt,

$$
\begin{aligned}
\left|v(t)-v_{n}(t)\right| & \leqq \sum_{v=2}^{\infty} \frac{\left|c_{v}\right|+\left|c_{v}^{(n)}\right|}{v !}(\varrho-\delta)^{v} \\
& \leqq k(1+\sqrt{2}) \sum_{v=2 n}^{\infty}\left(\frac{\varrho-\delta}{\varrho}\right)^{v}=k(1+\sqrt{2})\left(\frac{\varrho-\delta}{\varrho}\right)^{2 n} \frac{\varrho}{\delta} .
\end{aligned}
$$

Diese Differenz kann aber, indem man $n$ nur groß genug wählt, unabhängig von $t$ beliebig klein gemacht werden, und damit ist gezeigt, daß die Folge der Funktionen $v_{n}(t)$ mit wachsendem $n$ für $|t| \leqq \varrho-\delta$ gleichmäBig gegen $v(t)$ konvergiert.

\section{§ 2 .}

Wir kommen jetzt zu dem Beweise des in der Einleitung erwähnten funktionentheoretischen Hilfssatzes; das heißt, wir wollen zeigen, dab eine beliebige für $|t|<\varrho$ konvergente Potenzreihe

$$
v(t)=\sum_{\nu=0}^{\infty} b_{\nu} t^{\nu}=\sum_{\nu=0}^{\infty} \frac{c_{\nu}}{\nu !} t^{\prime} \quad(t==\sigma+i \tau),
$$

deren Koeffizientendeterminanten $C_{m}$ [vgl. Formel (5)] sämtlich positiv sind, sich in dem ganzen Streifen $-\varrho+\delta \leqq \sigma \leqq \varrho-\delta$ fortsetzen läßt.

Beweis: Da nach Voraussetzung die Potenzreihe $v(t)$ den Kreis $|t|=\varrho$ zum Konvergenzkreis besitzt, so ist nach bekannten Sätzen

$$
\left|b_{\nu}\right| \leqq \frac{k}{\left(\varrho-\frac{\delta}{4}\right)^{\nu}}, \quad\left|c_{\nu}\right| \leqq \frac{k \nu !}{\left(\varrho-\frac{\delta}{4}\right)^{\nu}},
$$

wo $k$ eine passend gewählte Konstante bedeutet.

Die Funktion $v(t)$ erfüllt daher die gleichen Voraussetzungen wie die Funktion (11) des $\S 1$, nur daß die Abschätzung ( $3^{\prime}$ ) an Stelle von (3) tritt. Es folgt daher aus den Ausführungen des vorigen Paragraphen, daB eine Folge von Funktionen

$$
v_{n}(t)=\sum_{x=1}^{n} M_{x}^{(n)} e^{\lambda_{x}^{(n)} t}
$$

existiert, die für $|z| \leqq \varrho-\delta$ gleichmäBig gegen $v(t)$ konvergiert. . Bie $\lambda_{n}^{(*)}$ ergeben sich als die wegen $C_{m}>0$ reellen einfachen Pole der Näherungsbrüche $K_{n}(z)$ des mit $S(z)$ assoziierten Kettenbruches $K(z)$, die $\boldsymbol{M}_{x}^{(n)}$ als die wegen $C_{m}>0$ positiven Residuen von $K_{n}(z)$ an den Polen $z=\lambda_{x}^{(n)}$.

Der Beweis unseres Hilfssatzes stützt sich auf einen oft angewandten Satz von Stieltjes über konvergente Folgen analytischer Funktionen ${ }^{14}$ ):

14) T. J. Stieltjes, Recherches sur les fractions oontinues, Ann. de la fac. des sciences de Toulouse 8 (1894), S. 50-61. 
Es sei eine unendliche Folge analytischer Funktionen $v_{n}(t)$ vorgelegt, die im Innern eines Bereiches $\Omega$ dem absoluten Betrage nach unterhalb einer festen gemeinsamen Schranke bleiben. Außerdem konvergiere in einem Teilbereiche $\Omega^{\prime}$ von $\Omega$ die Folge der Funktionen $v_{n}(t)$ gleichmäBig gegen eine analytische Funktion $v(t)$. Dann ist die analytische Funktion $v(t)$ in jedem ganz im Innern von $\Omega$ gelegenen Bereich $\Omega^{*}$ regulär, und die Funktionen $v_{n}(t)$ konvergieren in $\Omega^{*}$ gleichmäßig gegen die Funktion $v(t)$.

Um diesen Satz zum Beweise unseres Hilfssatzes anwenden zu können, ist nur noch nachzuweisen, daß die Funktionen $v_{n}(t)$ unseres Hilfsatzes in dem Bereiche $-\varrho+\frac{\delta}{2} \leqq \sigma \leqq \varrho-\frac{\delta}{2}$ dem absoluten Betrage nach unterhalb einer gemeinsamen festen Schranke $G$ bleiben. Dann folgt unser Hilfssatz unmittelbar aus dem Stjeltjesschen Satze.

Wir zeigen zunächst, daß die Funktionen $v_{n}(t)$ samt ihren Ableitungen $v_{n}^{(v)}(t)$ für rein imaginäre Werte $t=i \tau$, unterhalb einer festen Schranke bleiben, die nur von $v$, aber nicht von $n$ und $\tau$ abhängt. Wegen (9), (10), (13) und $\left(3^{\prime}\right)$ erbält man

$$
\begin{gathered}
v_{n}^{(2) 1)}(i \tau)=\sum_{x=1}^{n} M_{x}^{(n)}\left(\lambda_{x}^{(n)}\right)^{2 v} e^{\lambda_{x}^{(n)} i r} \\
\left|v_{n}^{(2 v)}(i \tau)\right| \leqq \sum_{x=1}^{n} M_{x}^{(n)}\left(\lambda_{x}^{(n)}\right)^{2 \nu}=c_{2 v}^{(n)} \leqq k \frac{(2 v) !}{\left(e-\frac{\delta}{4}\right)^{q \nu},}
\end{gathered}
$$

da $M_{x}^{(n)}>0$ ist. Ebenso findet man

$$
\left|\boldsymbol{v}_{n}^{(n \gamma+1)}(i \tau)\right| \leqq \sum_{\kappa=1}^{n} M_{\kappa}^{(n)}\left|\lambda_{\kappa}^{(n)}\right|^{2 \gamma+1} ;
$$

aus der Schwarzechen Ungleichheitsbeziehung urd der Abschätzung (14) in Verbindung mit $\left(3^{\prime}\right)$ ergibt sich endlich

$$
\begin{aligned}
& \left|v_{n}^{(8 v+1)}(i \tau)\right| \leqq \sqrt{\sum_{x=1}^{n} M_{n}^{(n)}\left(\lambda_{x}^{(n)}\right)^{2 v+2} \sum_{x=1}^{n} M_{x}^{(n)}\left(\lambda_{x}^{(n)}\right)^{2 v}} \\
& \leqq \sqrt{c_{2 v+1} c_{8 \nu}} \leqq i \sqrt{2} \frac{(2 v+1) !}{\left(e-\frac{d}{4}\right)^{2 v+1}} \text {. }
\end{aligned}
$$

Es sei jetzt $t=\sigma+i t$ ein bellebiger- P'tinkt des Bereiches $-\varrho+. \frac{\delta}{2} \leqq \sigma \leqq \varrho-\frac{\delta}{2}$, dann erhält man, wenn man $v_{n}(\sigma+i \tau)$ in eine Taylorsche Reihe entwickelt, auf Grund der A'bschätzungen (15) und (16) 


$$
\begin{aligned}
& v_{1,}(t)-\mid \sum_{r=0}^{\infty} \frac{\sigma^{\prime}}{i !} v_{n}^{n}(i \tau) \leqq \sum_{r=0}^{x} \frac{\left(g-\frac{\partial}{2}\right)^{\prime}}{\nu^{\prime}} v_{n}^{(\nu)}(i \gamma) \\
& \leqq k \sqrt{2} \sum_{i=0}^{x}\left(\frac{e-\frac{\delta}{2}}{a-\frac{d}{4}}\right)^{\prime}-k v^{\prime} \overline{2}^{4} \underline{\delta} \text {. }
\end{aligned}
$$

Somit bleiben also die Funk tionen $v_{n}(t)$ im Streifen $-\theta+\frac{\delta}{2} \leqq \sigma \leqq \theta-\frac{\delta}{2}$ dem absoluten Betrage nach unterhalb einer festen von $n$ und $t$ unabhängigen Schranke $G=k \sqrt{2}{ }_{\delta}^{4}-\delta$, konvergieren also nach dem Satze von Stieltjes gleichmäßig in dem Bereiche $-\varrho+\delta \leqq \sigma \leqq \varrho-\delta,|\tau| \leqq R$ für jedes noch so große positive $R$ gegen die dort analytische Funhtion $v(t)$.

Aus der Abschätzung

$$
\left|v_{1 i}(t)\right| \leqq G
$$

folgt endlich für denselben Bereich auch

$$
v(t) \mid \leqq G
$$

Damit sind alle Behauptungen des Hilfssatzes bewiesen.

Zusatz: Sind auper den aus den Koeffizienten $c_{\nu}=\nu ! b_{\nu}$. gebildeten Determinanten' $C_{m}$ auch sämtliche Determinanten

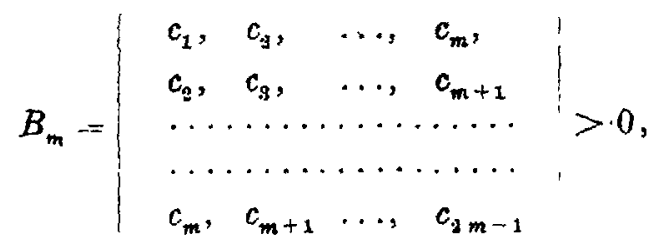

80 existiert, wenn wieder mit $\varrho$ der Konvergenzradius der Potenzreihe (11) bezeichnet wird, die durch diese Reihe dargestellte Funktion, $v(t)$ in dor ganzen. Halbebene $\sigma \leqq \varrho-\delta$ und bleibt dort kleiner als eime Jeste wart unabhängige Große.

Obgleich im folgenden von diesem Satze kein Gebrauch gemacht wird, soll hier sein Beweis kurz angegeben werden, da er an sich vonn Tntaresise exscheint.

Beweis: Wie beim vorigen Beweise gezeigt wurde, läßt sich wegen der Voraussetzung $C_{m}>0$ für alle $m$ in dem Streifen $-\varrho+\delta \leqq \sigma \leq \varrho-\delta$ die Funktion $v(t)$ als Grenzwert der konvergenten Folge ton Tunktionen

$$
v_{n}(t)=\sum_{x=1}^{n} M_{x}^{(n)} e^{i_{\kappa}^{(n)} t}
$$


darstellen. Nun folgt aber nach bekannten Sätzen ${ }^{14}$ ) aus der Voraussetzung $B_{m}>0$ für alle $m$, daß die Näherungsbrüche $K_{n}(z)$ des mit der Potenzreihe $S(z)$ assoziierten Kettenbruches nur für positive Werte von $z$ Pole haben können. Da ferner, wie oben gezeigt wurde, die Stellen $z=\lambda_{x}^{(n)}$ gerade die Pole von $K_{n}(z)$ sind, so ist $\lambda_{\kappa}^{(n)}>0$ für alle $n$ und alle $\%$. Man erbält also für $\sigma \leqq 0$ wegen $M_{\%}^{(n)}>0$

$$
\left|v_{n}(t)\right| \leqq \sum_{x=1}^{n} M_{x}^{(n)} e^{\sigma \lambda_{x}^{(n)}} \leqq \sum_{x=1}^{n} M_{*}^{(n)}=c_{0}
$$

- Die $v_{n}(t)$ bleiben also in der ganzen Halbebene $\sigma \leqq 0$ unterhalb einer festen von $n$ und $t$ unabhängigen Schranke, und daraus folgt die Behauptung des Zusatzes unmittelbar unter Benutzung des Stieltjesschen Satzes.

\section{$\S 3$.}

Der Nachweis, da $\beta^{*}$ der mit der Potenzreihe $S(z)$ assoziierte Kettenbruch $K(z)$ für $y \geqq \delta$ gleichmäßig gegen die Funktion

$$
f(z)=\int_{0}^{-i \infty} v(t) e^{-t z} d t
$$

konvergiert, bereitet nun keine Schwierigkeiten mehr.

Zunächst folgt aus der Abschätzung (18) für $v(t)$, die längs des gesamten Integrationsweges des Integrals (19) gilt, daB das Integral (19) für $y \geqq \delta$ absolut und gleichmäßig konvergiert.

Andereneits war nach Formel (8)

$$
K_{n}(z)=\int_{0}^{-i \infty} v_{n}(t) e^{-t z} d t
$$

Es soll nunmehr gezeigt werden, dab zu den beiden vorgegebenen beliebig kleinen Zahlen $\varepsilon$ und $\delta$ eine ZahI $N$ von der Beschaffenheit bèstimmt werden kann, daß für $y \geqq \delta$ und alle $n \geq N$

$$
\left|f(z)-K_{n}(z)\right| \leqq \varepsilon
$$

wird. Es ist

$\left.\left|f(z)-R_{n}(z)\right| \leqq \int_{0}^{-R} \mid v(i \tau)-v_{n}(i \tau)\right\} e^{\tau \delta} d \tau+\int_{-R}^{\infty}\left(|v(i \tau)|+\left|v_{n}(i \tau)\right|\right) e^{\tau d} d \tau$

Zunächst werde $\boldsymbol{R}$ so groß gewählt, daß.

$$
\int_{-R}^{\infty} e^{x \delta} d \tau=\frac{e^{-R \delta}}{\delta} \leqq \frac{\varepsilon}{3 G}
$$

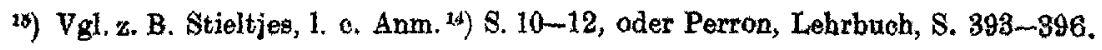


wird, wobei $R$ nur von $\varepsilon$ und $\delta$ abhängt. Danm wird auf Grund der Abschätzungen (17) und (18)

$$
\int_{-R}^{-\infty}\left(|v(i \tau)|+\left|v_{n}(i \tau)\right|\right) e^{r d} d \tau \leqq \frac{2}{3} \varepsilon .
$$

Jetzt wähle man eine hinreichend große Zahl $N=N(\varepsilon, \delta)$, die nur von $c$ und $\delta$ abhängt von der Beschaffenheit, daß für $0 \geqq \tau \geqq-R$

ist; dann erhält man

$$
\left|v(i \tau)-v_{n}(i \tau)\right| \leqq \frac{\varepsilon \delta}{3}
$$

$$
\int_{0}^{-R}\left|v(i \tau)-v_{n}(i \tau)\right| e^{r \delta} d \tau \leqq \frac{\varepsilon}{3} .
$$

Nunmehr folgt (20) für $n \geqq N=N(\varepsilon, \delta)$ aus (21) und (22).

Entsprechend zeigt man, daß für $y \leqq-\delta$ die Näherungsbrüche $K_{w}(z)$ gleichmäßig gegen die Funktion

konvergieren.

$$
f^{*}(z)=\int_{0}^{i x} v(t) e^{-t z} d t
$$

EB ist für $y \geqq \delta$, wie aus der Partialbruchzerlegung (7) für $K_{n}(z)$ unmittelbar heryorgehty.

$$
K_{n}(x-i y)=\bar{K}_{n}(x+i y),
$$

wenn mit $\bar{K}_{n}(x+i y)$ der zu $K_{n}(x+i y)$ konjugiert komplexe Wert bezeichnet wird. Aus (29) folgt aber

$$
f^{*}(x-i y)=\bar{f}(x+i y) \text {. }
$$

Damit ist der in der Einleitung angegebene Konvergenzsatz für Kettenbrüche vollständig bewiesen.

$\S 4$.

Aus den Sätzen des Kap. III der Grommerschen Dissertation ${ }^{18}$ ) folgt, daß sich $f(z)$ für $y \geqq \delta$ durch ein Stieltjessches Integral.

$$
f(z)=\int_{-\infty}^{+\infty} \frac{d \Phi(u)}{z-u}
$$

darstellen läßt, wobei mit $\Phi(u)$ eine reelle, nirgends abnehmende Frrmktion von ' $u$ bezeichnet ist. 
Fiür $y<0$ findet man wegen $f^{*}(x+i y)=\bar{f}(x-i y)$ gleichfalls

$$
f^{*}(z)=\int_{-\infty}^{+\infty} \frac{d \Phi(u)}{z-u} .
$$

Die Darstellungen (24) und (25) lassen erkennen, wann $f^{*}(z)$ die analytische Fortsetzung von $f(z)$ ist: nämlich dann und nur dann, wenz ein Intervall $a \leqq u \leqq b$ existiert, in dem $d \Phi(u)$ identisch verschwindet.

Denn in diesem Falle lassen sich die Integrale (24) bzw. (25) in der Form schreiben

$$
\begin{aligned}
f(z)=\int_{-\infty}^{a} \frac{d \Phi(u)}{z-u}+\int_{b}^{\infty} \frac{d \Phi(u)}{z-u} & \text { für } y>0, \\
f^{*}(z)=\int_{-\infty}^{a} \frac{d \Phi(u)}{z-u}+\int_{b}^{\infty} \frac{d \Phi(u)}{z-u} & \text { für } y<0:
\end{aligned}
$$

Da ferner die Integrale (26) in den Vertikalstreifen $a+\delta \leqq x \leqq b-\delta$ gleichmäßig konvergieren und in diesem Gebiete reguläre analytische Funktionen . darstellen, so erweist sich $f^{*}(z)$ als diejenige Funktion, die man. erhält, wenn man $f(z)$ längs des Intervalles $a+\delta \leqq x \leqq b-\delta$ über die Ebene der Zahlen mit positivem Imaginärteil hinaus fortsetzt.

Die angegebene Bedingung ist aber auch notwendig dafür, daB $f(z)$ und $f^{*}(z)$ dieselbe analytische Funktion darstellen; denn nach einem Satz des Flerrn Grommer ${ }^{27}$ ) folgt aus

$$
\lim _{y=0}\left[f(x+i y)-f^{*}(x-i y)\right]=\varphi(x) \quad \text { für } a \leqq x \leqq b,
$$

daß $\Phi(u)$ in dem Intervall $a \leqq u \leqq b$ differentiierbar, und zwar daß

ist.

$$
\frac{d \Phi(x)}{d x}=\frac{\varphi(x)}{2 \pi i}
$$

Ist nun $f^{*}(x-i y)$ für $y>0$ die analytische Fortsetzung von $f(x+i y)$ längs des Intervalles $a \leqq x \leqq b$, so ist in diesem Intervall. $\varphi(x)=0$, also auch $d \Phi(x)=0$. W. z. b. w.

Zum Schlusse noch eine kurze Bemerkang über eine letzte, vierte Daxstellung der Funktion $f(z)$.

Da $f(z)$ die durch Borelsche Summation der Reihe $S(z)$ gebildete 
Funktion ist, so läßt sie sich nach Sätzen der Herren Pincherle ${ }^{18}$ ), Horn ${ }^{19}$ ) und Nörlund ${ }^{20}$ ) in eine Fakultätenreihe entwickeln von der Form

$$
f(z)=\sum_{\nu=1}^{\infty} \frac{(i \omega)^{\nu} a_{\nu} \nu !}{z(z+i \omega)(z+2 i \omega) \ldots(z+(\nu-1) i \omega)} .
$$

Hierbei ist $\omega$ eine pasitive Konstante, die sich nach einem Satze von Herrn Nörlund ${ }^{21}$ ) so bestimmen läßt, daß die Reihe (27) in der Halbebene $y \geqq \delta$ gleichmäßig konvergiert.

Die $a_{v}$ sind in einfacher Weise durch die $c_{r}$ bestimmt; es ist nämlich

$$
\begin{aligned}
a_{y} & =\sum_{x=1}^{v} \gamma_{\gamma_{\varkappa}} c_{x}, \\
c_{\nu} & =\sum_{\nu=1}^{\gamma} \Gamma_{\nu_{\alpha}} a_{\lambda} .
\end{aligned}
$$

Hierbei sind die $\gamma_{\nu *}$ bzw. $\Gamma_{\psi *}$ Konstanten, die nur von $\omega$, aber nicht von der speziellen Wahl der $c_{v}$ bzw. $a_{v}$ abhängen und für die sich leicht explizite Formeln angeben lassen.

5. Hiermit sind für die Funktion $f(z)$ vier für $y \geqq \delta$ gleichmäßig konvergente Darstellungen gefunden: erstens durch den Kettenbrach $K(z)$ der Formel (1), zweitens durch das Borelsche Integral (4), drittens durch das Stieltjessche Integral (24), viertens durch die Fakultätenreihe (27): .Endlich läßt sich $f(z)$ noch durch die. Potenzreihe $S(z)$ im Winkelraum $\delta \leqq \arg z \leqq \pi-\delta$ asymptotisch darstellen.

18) Pincherle, Sur les fonctions déterminantes, Ann. scient. de l'éc. norm. sup. 22 (1905), S. 1-68, vgl. insbes. S. 50-57.

19) J. Horn, Fakultätenreihen in der Theorie der linearen Differentialgleichungen, Math. Ann. 71 (1912), S. 510-532.

$\left.{ }^{20}\right)$ Nörlund, Sur les séries de facultés, Acta math. 37 (1914), S. 327-387.

21) 1. o. Anm. ${ }^{19}$ ) S. 371.

(Angenommen November 1919.) 